\title{
6. Das Wissensportal der ETH-Bibliothek - ein Fallbeispiel für User-Centered-Design
}

\section{Arlette Piguet}

\section{Einführung}

Die Bibliothek der Eidgenössischen Technischen Hochschule Zürich hat seit Mitte des Jahres 2010 einen neuen Internetauftritt (www.library.ethz.ch): Das „Wissensportal“ der ETH-Bibliothek bietet über einen Single-Point-of-Access einen neuartigen Zugang zu den heterogenen Informationsressourcen und Dienstleistungen der technisch-naturwissenschaftlich ausgerichteten Hochschulbibliothek. Es integriert diverse elektronische Informationsangebote in einem zentralen Rechercheinstrument und bindet dieses nahtlos in die Website mit allen Dienstleistungen der ETH-Bibliothek ein. Das neue Portal war bei den Kunden von Beginn an sehr beliebt und ist heute nicht mehr wegzudenken.

Die Qualitätsansprüche an das neue Angebot wurden hoch angesetzt, wobei die Bedürfnisse des heterogenen Zielpublikums klar im Zentrum standen. Ein ansprechendes Design und eine intuitive Benutzung des Wissensportals gehörten zu den wichtigsten Projektzielen.

In diesem Beitrag werden die einzelnen Schritte skizziert, die nötig waren, um ein möglichst optimal auf die Bedürfnisse der Nutzer-Community abgestimmtes Portal zu erarbeiten. Doch wer sind diese Nutzer? Welche spezifischen Bedürfnisse an das Online-Angebot einer Hochschulbibliothek haben sie und wie nutzen sie dieses? Wie lassen sich diese Anforderungen überhaupt ermitteln, und wie können die identifizierten Bedürfnisse mit einem adäquaten Design und benutzerfreundlichen Funktionalitäten in die Praxis umgesetzt werden?

\section{User-Centered-Design: ein kurzer Blick in die Theorie}

Der Ansatz des User-Centered-Designs geht unter anderem auf Arbeiten von Gould und Lewis zurück ${ }^{1}$. Bei dieser Herangehensweise stehen die Benutzerbedürfnisse sowie das Design für die intuitive und einfache Bedienung eines Produktes - das die Interaktion von Mensch und Maschine erlaubt - im Zentrum².

1 Vgl. hierzu etwa: Gould und Lewis (1985).

2 Weitere Arbeiten zum Thema vgl. etwa: Normann (1998) oder Jordan (1998). 
Das Produkt kann beispielsweise eine Website sein, zu deren benutzerfreundlichen Bedienung auch eine auf den ersten Blick erfassbare Struktur sowie dem Medium angepasste Texte gehören. Im Bibliotheks- und Informationsbereich hat das User-Centered-Design auch große Relevanz für Rechercheinstrumente.

Für die Durchführung des benutzerorientierten Gestaltungsprozesses haben sich mehrere Evaluationsmethoden durchgesetzt, die insbesondere auch vom langjährigen Usability-Experten Jakob Nielsen mitgeprägt wurden. Eine Hilfestellung für die Entwicklung von Software - dazu gehören auch Websites bietet beispielsweise aber auch die Norm ISO 13407 aus dem Jahr 1999³.

Die Beteiligung der zukünftigen Nutzer im gesamten Entwicklungsprozess des Produktes ist ein wesentlicher Kern zahlreicher Verfahren. Die Kenntnis und die Orientierung an den Aufgaben, Zielen sowie Fähigkeiten und Grenzen der potenziellen Nutzer sind von Beginn an unumgänglich. Der gesamte Entwicklungsprozess verläuft denn auch kooperativ und iterativ: Erste auf der Basis vom Nutzeranalysen und Interviews erarbeitete Prototypen - allenfalls lediglich auf Papier skizziert - werden mit potenziellen Nutzern getestet, auf Basis der gewonnenen Erkenntnisse adaptiert und allenfalls erneut getestet. Die Anzahl der Zyklen hängt im Allgemeinen von der Komplexität des Anwendungsgebietes ab. Mit diesem Entwicklungsprozess kann die Qualität des Interface deutlich gesteigert werden.

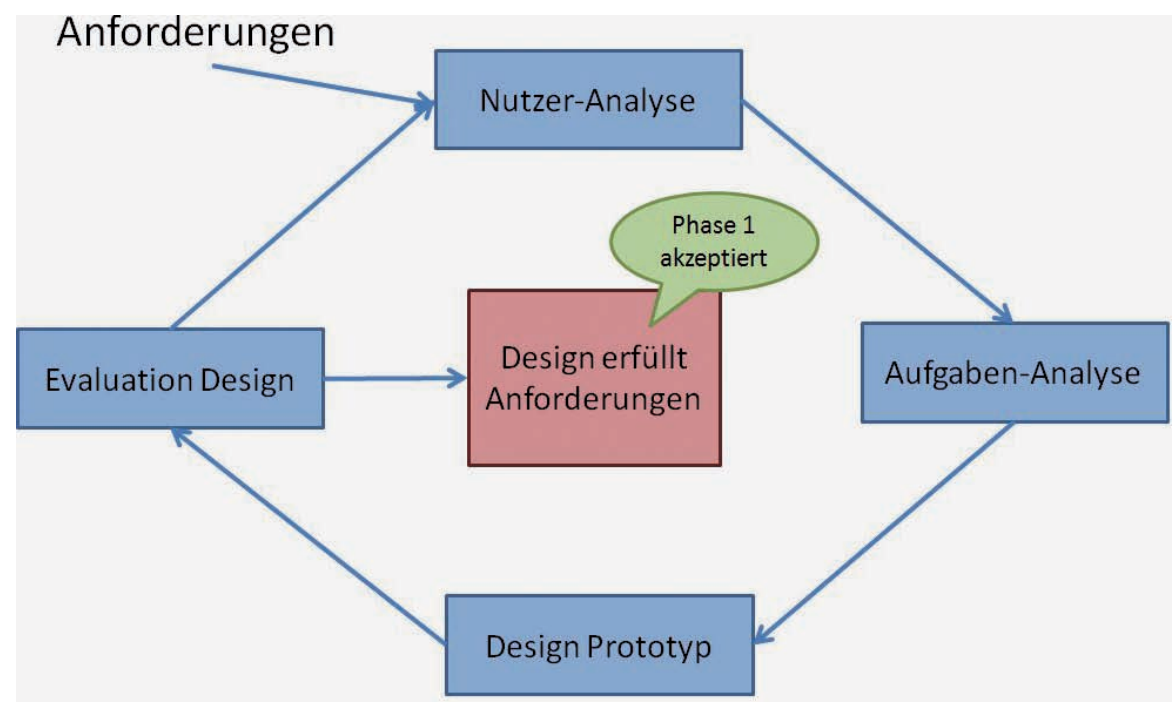

Abb. 1: Der User-Centered-Design-Prozess nach ISO 13407; angelehnt an eine Präsentation von Hauri, Ergonomie \& Coaching GmbH (http://www.chauri.ch/)

3 Deutsche Fassung: EN_ISO_13407: 1999. 
Im Gegensatz zu den subjektiven Evaluationsmethoden, die unmittelbar an die Beurteilung des Benutzers anknüpfen und somit eher „weiche Daten“ liefern, werden bei den objektiven Evaluationsmethoden quantitative Daten erhoben. „Zwischen den subjektiven und objektiven Methoden liegen die analytische (leitfadenorientierte) Evaluation durch Experten und die empirische Evaluation (usability assessed by testing the interface with real users). " " Die Wahl einer spezifischen Evaluationsmethode hängt von mehreren Fragestellungen ab, u.a. vom Ziel der Evaluation, der Information die gesammelt werden soll oder auch von den verfügbaren Ressourcen ${ }^{5}$. Oberstes Ziel bleibt es immer, die Software an die Bedürfnisse der Benutzer anzupassen.

\section{Die ETH-Bibliothek - eine innovative Dienstleistungseinrichtung}

Die ETH-Bibliothek ist die zentrale Hochschulbibliothek für die ETH Zürich. Als grösste Bibliothek der Schweiz mit einem Bestand von rund 7,6 Mio. Einheiten nimmt sie auch die Aufgabe eines nationalen Zentrums für naturwissenschaftliche und technische Information wahr. Zu den Forschungsschwerpunkten der ETH Zürich gehören die Ingenieurwissenschaften, die Architektur, die systemorientierten Wissenschaften sowie Mathematik und die Naturwissenschaften. Die ETH-Bibliothek nimmt für diese Fachgebiete somit auch die Rolle einer Spezialbibliothek ein.

Naturgemäss zählen die Angehörigen der Hochschule, also Wissenschaftler sowie Studierende, zum primären Zielpublikum der ETH-Bibliothek ${ }^{6}$. Als Institution mit öffentlichem Auftrag versorgt sie aber auch Kunden aus der Industrie sowie die wissenschaftlich interessierte Öffentlichkeit mit Informationen. Da die ETH-Bibliothek über ein umfassendes Informationsangebot verfügt, benutzen selbstverständlich auch Studierende sowie das wissenschaftliche Personal von anderen Schweizer Bildungseinrichtungen die ETH-Bibliothek.

Besondere Schwerpunkte liegen im Bereich elektronischer Informationsangebote sowie in der Entwicklung und Realisierung innovativer Dienstleistungsangebote. Zum aktuellen Online-Angebot zählen der Katalog sowie eine sehr umfassende Anzahl an lizenzierten Fachdatenbanken, elektronischen Zeitschriften und zunehmend auch an Büchern in elektronischer Form. Darüber hinaus digitalisiert die ETH-Bibliothek seit mehreren Jahren schweizerische Zeitschriften, alte Drucke sowie Bilder und stellt diese Materialien kostenlos weltweit über geeignete Plattformen online zur Verfügung. Der bereits seit rund zehn Jahren angebotene Dokumentenserver wird seit kurzem durch eine

4 So: Hegner (2003).

5 Vgl. hierzu: ebenda.

6 Die ETH Zürich zählt rund 16000 Studierende, 400 Professoren sowie über 7000 wissenschaftliche Mitarbeitende und administratives Personal. 
Hochschulbibliographie ergänzt. Die Heterogenität dieses umfassenden Angebotes hat auch eine Kehrseite: die Benutzung ist relativ komplex und vor allem für gelegentliche Nutzer ist es nicht einfach, sich im Angebot zurechtzufinden.

Es ist davon auszugehen, dass insbesondere im Wissenschaftsbereich in den nächsten Jahren die Informationsversorgung noch verstärkt und allenfalls sogar ausschließlich auf elektronischem Weg erfolgen wird. Hinzu kommt, dass die Kunden im Zeitalter der leistungsfähigen Suchmaschinen ganz andere Ansprüche an die Benutzerfreundlichkeit von Katalogen und weiteren Informationsangeboten haben als früher. Nicht zuletzt aus diesem Grund wollte die ETH-Bibliothek einen Paradigmenwechsel vollziehen: Die bisherige datenbankbasierte Suche im Katalog sollte durch eine suchmaschinenbasierte Abfrage ersetzt werden, wie man sie von gängigen Suchmaschinen her gewohnt ist. Auch das Idealziel eines „Single-Point-of-Access“ für alle online angebotenen Informationsressourcen sowie für die weiteren Dienstleistungen der ETHBibliothek sollte im Rahmen eines Projektes soweit möglich realisiert werden.

Dass der Weg in die skizzierte Richtung korrekt ist, untermauert auch eine im Jahr 2007 von der ETH-Bibliothek durchgeführte qualitative Expertenbefragung zum Umgang mit Literatur und Informationsressourcen in Forschung und Lehre an der ETH Zürich ${ }^{7}$. Die Nutzung verlagert sich zunehmend auf elektronische Angebote, wobei die Wissenschaftler häufig das Problem äußern, sich in der wachsenden Menge an Informationen zunehmend nicht mehr zurechtzufinden. Damit die ETH-Bibliothek ihre Rolle als Informationsanbieter und -vermittler auch in Zukunft wahrnehmen kann, muss der Zugang zu relevanten Informationen somit möglichst einfach und intuitiv sein.

\section{Projektziel und Rahmenbedingungen}

Ziel des Projektes Wissensportal war es somit, den Kunden der ETH-Bibliothek einen wesentlich vereinfachten Online-Zugang zum Informations- und Dienstleistungsangebot über einen Single-Point-of-Access bereitzustellen. Bereits auf der ersten Ebene der Website sollte eine intuitive, schnelle und integrierte Suche in den Katalogdaten sowie in weiteren Informationsressourcen möglich sein. Auch die vielfältigen Dienstleistungen der ETH-Bibliothek sollten benutzerfreundlich präsentiert werden. Darüber hinaus sollte das neue Portal attraktiv sein und mit einem ansprechenden Design ein zeitgemässes Image der ETHBibliothek vermitteln. Die Anforderungen an den neuen Internetauftritt beinhalteten also wichtige Aspekte, die im Kontext des User-Centered-Designs relevant sind. Um den Bedürfnissen der Nutzer des künftigen Informationsportals möglichst weit entgegenzukommen, wurde für die Umsetzung der gestellten Anforderungen der oben skizzierte User-Centered-Design-Prozess verfolgt.

Vgl. hierzu: Ramminger und Graf (2007). 
Das Großprojekt dauerte etwas mehr als zwei Jahre. Der auf das Portal abgestimmte User-Centered-Design-Prozess erstreckte sich über mehrere Monate.

Das Projekt wurde nach einem in der ETH-Bibliothek etablierten standardisierten Projektverfahren abgewickelt ${ }^{8}$. Für die Bearbeitung der hohen Projektanforderungen war die Zusammenstellung eines professionellen Teams aus Bibliothekaren, technischem Personal, Designern sowie der für das Marketing verantwortlichen Person essenziell. Das Subteam Website war für das Design, ein zweites kleines Subteam für die Inhalte der Website verantwortlich. Professionelle Unterstützung wurde von einem Usability-Experten und einer auf Web-Design spezialisierten Firma in Anspruch genommen.

Zusätzlich zur Qualitätsorientierung war auch die Orientierung an international anerkannten technischen Standards von entscheidender Bedeutung für das Vorhaben.

Bereits früh im Projekt wurde entschieden, für die Realisierung der Suchfunktionalitäten ein kommerzielles Produkt zu nutzen und dieses auf die eigenen Bedürfnisse anzupassen. Der Aufwand für eine Eigenentwicklung auf Basis einer Open-Source-Suchmaschine wurde aus zeitlichen, personellen wie auch aus finanziellen Gründen verworfen. Nach einem längeren Evaluationsprozess fiel die Wahl auf das Produkt Primo der Firma ExLibris. Dieses Suchinstrument erlaubt die Suche, die Anzeige und das Delivery von heterogenen Informationsressourcen. Für die Realisierung der integrierten Suche müssen die Metadaten naturgemäß normalisiert werden. Diese Arbeit wurde im Rahmen eines Teilprojektes realisiert.

Die ETH-Bibliothek entschied sich darüber hinaus, die Datenbank DADS ${ }^{9}$ der Technischen Universität Dänemark (DTU) an Primo anzubinden ${ }^{10}$. Diese Datenbank beinhaltet mehrere Millionen Metadatensätze von Artikeln wichtiger wissenschaftlicher Zeitschriftenverlage. Mittlerweile sind auch die Daten von Web of Science integriert.

Das mit Primo standardmässig ausgelieferte Design kann über Änderungen in den Cascading-Style-Sheets (CSS) in begrenztem Ausmass auf die eigenen Bedürfnisse angepasst werden. Weiterführende Anpassungen können über Änderung in den Java Server Pages bzw. HTML-Files realisiert werden. Für die Integration der Websiteinhalte war es erforderlich, parallel dazu ein ContentManagement-System (CMS) zu implementieren ${ }^{11}$.

8 Das Verfahren orientiert sich an der schweizerischen Projektführungsmethode HERMES, der Methode für die einheitliche und strukturierte Durchführung von Projekten der Informations- und Kommunikationstechnik (IKT). Vgl. hierzu: http://www.hermes.admin. $\mathrm{ch} /$.

9 DADS: Digital Article Database Service.

10 Über das sogenannte „Deep-Search-Konzept“ können in Primo auch externe Datenbanken angebunden und gleichzeitig mit den lokalen Daten durchsucht werden.

11 Das System eZ Publish erfüllte die definierten Anforderungen am besten. 
Die Ausgangslage für die Präsentation der heterogenen Inhalte war sichtlich komplex. Das Design für eine einfache Navigation und Nutzung musste folglich für diverse Aspekte entwickelt werden:

- Suchfunktionen für die lokalen und externen Metadaten sowie für die Inhalte der Website

- Präsentation der Suchergebnisse mit Funktionen für die weitere Einschränkung

- Funktionalitäten für den Zugriff auf die recherchierten Inhalte

- Präsentation der Informationen zu allen Dienstleistungen der ETH-Bibliothek

- Navigation durch die gesamten Inhalte der Website

Ziel war es nun, das Suchmaschinenprogramm Primo der Firma ExLibris hinsichtlich Design und Funktionalitäten so weit in das CMS der ETH-Bibliothek $\mathrm{zu}$ integrieren, dass die Suchmaschine von aussen gesehen als fester Bestandteil der Website erscheint.

\section{Schrittweises Vorgehen}

\section{Website-Analyse}

Von Anfang an war klar: Das neue Portal sollte kein Aufguss der alten Website sein. Es sollte andersartig und auch „besser“ werden. Trotz oder gerade wegen dieser Anforderung an das Wissensportal schien es angezeigt, in einem ersten Schritt die alte Website der ETH-Bibliothek auf ihre Unzulänglichkeiten hin zu analysieren sowie Ideen von attraktiven Websites von „Konkurrenzinstitutionen“ zu sammeln.

Die Ist-Analyse der Vorgänger-Website machte - größtenteils bereits bekannte - Mängel der bereits im Jahr 1999 konzipierten Website deutlich. Hierzu zählten beispielsweise zu lange Texte, teilweise eine zu stark an die bibliothekarische Terminologie angelehnte Sprache oder die nicht mehr konsistente Struktur und Navigation. Diese war selbstverständlich auch bedingt durch immer neue Informationsdienstleistungen, die im Verlauf der Zeit in die bestehende Website eingefügt wurden. Die Analyse der Log-Files einzelner Seiten gab zusätzlich darüber Auskunft, welche Angebote verhältnismässig wenig benutzt und allenfalls prominenter platziert werden müssen.

Darüber hinaus wurden anhand einer Checkliste auch die Websites der Bibliotheken von vergleichbaren, ebenfalls renommierten technisch-naturwissenschaftlich ausgerichteten Hochschulen ${ }^{12}$, aber auch von nicht-bibliothe-

12 Dies waren u.a. die MIT Libraries (http://libraries.mit.edu/), die University of Toronto Libraries (http://main.library.utoronto.ca/) sowie die University of California Library (http://www.library.ucla.edu/). 
karischen Institutionen analysiert. Beurteilt wurde der Gesamteindruck, die Zielgruppenorientierung (Sprache, Design u.a.), das Visual Design (Layout, Farben u.a.), die Informationsarchitektur (Struktur, Navigationswege u.a.), die Benutzerführung (Konsistenz, Navigationselemente u.a.), die Inhalte (Übersichtlichkeit, Umfang, Schreibstil u.a.) sowie die Technik (Schnelligkeit, Fehlermeldungen u.a.).

Die aus Sicht der ETH-Bibliothek positiven sowie die weniger gelungenen Aspekte wurden festgehalten und während des gesamten Designprozesses für das eigene Portal immer wieder als Referenz für die Veranschaulichung von Teilaspekten herangezogen.

\section{Workshops}

Der eigentliche User-Centered-Design-Prozess wurde in mehreren Teilschritten abgearbeitet:

\section{Interner Workshop (Benutzergruppen, Szenarien)}

Ein erstes Ziel war es, die potenziellen Nutzer der zukünftigen Website festzulegen und vor allem auch deren Charakteristika zu identifizieren. Hierzu wurden in einem ersten noch bibliotheksinternen Workshop alle infrage kommenden Benutzergruppen des zukünftigen Wissensportals aufgelistet, charakterisiert und priorisiert. Grundlage war der durch den Steuerungs- und Fachausschuss des Projektes definierte Katalog der Zielgruppen der ETH-Bibliothek:

- Erfahrene Forschende

- Lehrende

- Masterstudierende, Jungforscher

- Studienanfänger, Bachelorstudierende

- Alumni, technisch interessierte Wissenschaftler

- Historisch interessierte externe Wissenschaftler

- Firmenkunden (Sonderfall)

- Öffentlichkeit

- Schüler

- ETH-Mitarbeitende (administrative und technische Angestellte)

Das Nutzerspektrum ist also nicht nur breit, die einzelnen Gruppen stellen zusätzlich unterschiedlichste Anforderungen. Dieses Faktum wurde für die Konzipierung des Designs mit intuitiver Bedienung als wichtiger Faktor identifiziert. 


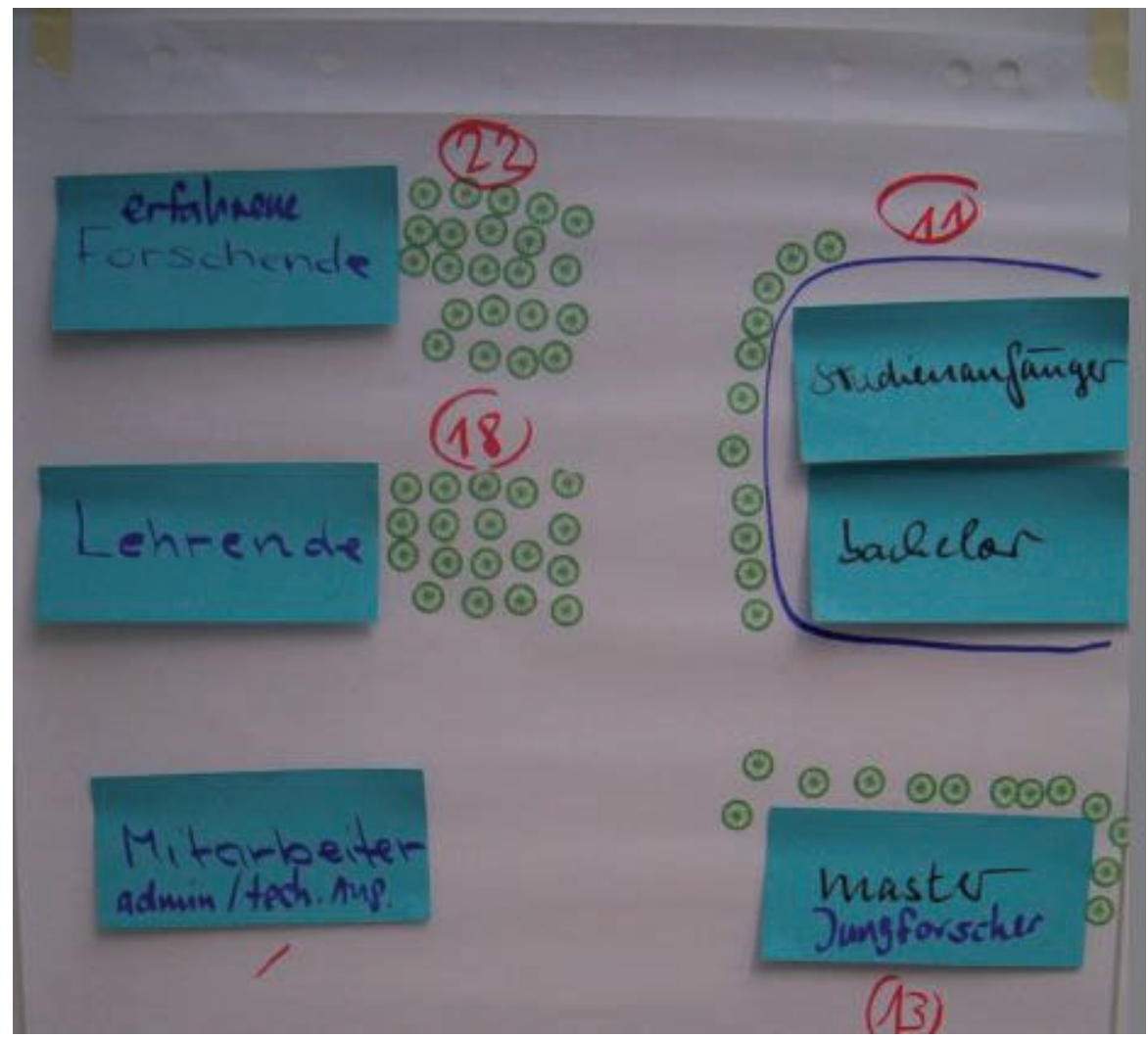

Abb. 2: Potenzielle Nutzer des zukünftigen Wissensportals; Skizze mit Bewertung der Wichtigkeit (grüne Punkte) aus dem Workshop

Von den identifizierten Nutzergruppen wurden anschließend Benutzerprofile entwickelt, sogenannte Personas ${ }^{13}$, und als (erfundene) realistische Personenbeschreibungen dokumentiert:

- Name, Alter, Beruf

- Ausbildung, Vorkenntnisse

- Ziele, Nutzungsziele

- Aufgaben, Tätigkeiten

- Vorlieben, Spezielles, Abneigungen, Motto...

Ziel war, dass sich die Projektmitarbeitenden ein einprägsames Bild von den jeweiligen Benutzertypen machen konnten. Bei dieser Aufgabe hatten die Workshopteilnehmer sichtlich Spass, was sich auch positiv auf das Ergebnis auswirkte.

13 Vgl. hierzu: Cooper (1999). 
Das Wissensportal der ETH-Bibliothek - ein Fallbeispiel für User-Centered-Design 127

In einem zweiten Schritt wurden für die wichtigsten Nutzergruppen mögliche Nutzungsszenarien erarbeitet. Aufgrund der Diskussion solcher Szenarien entstanden erste wichtige Erkenntnisse über die grundlegenden Anforderungen an die Website.

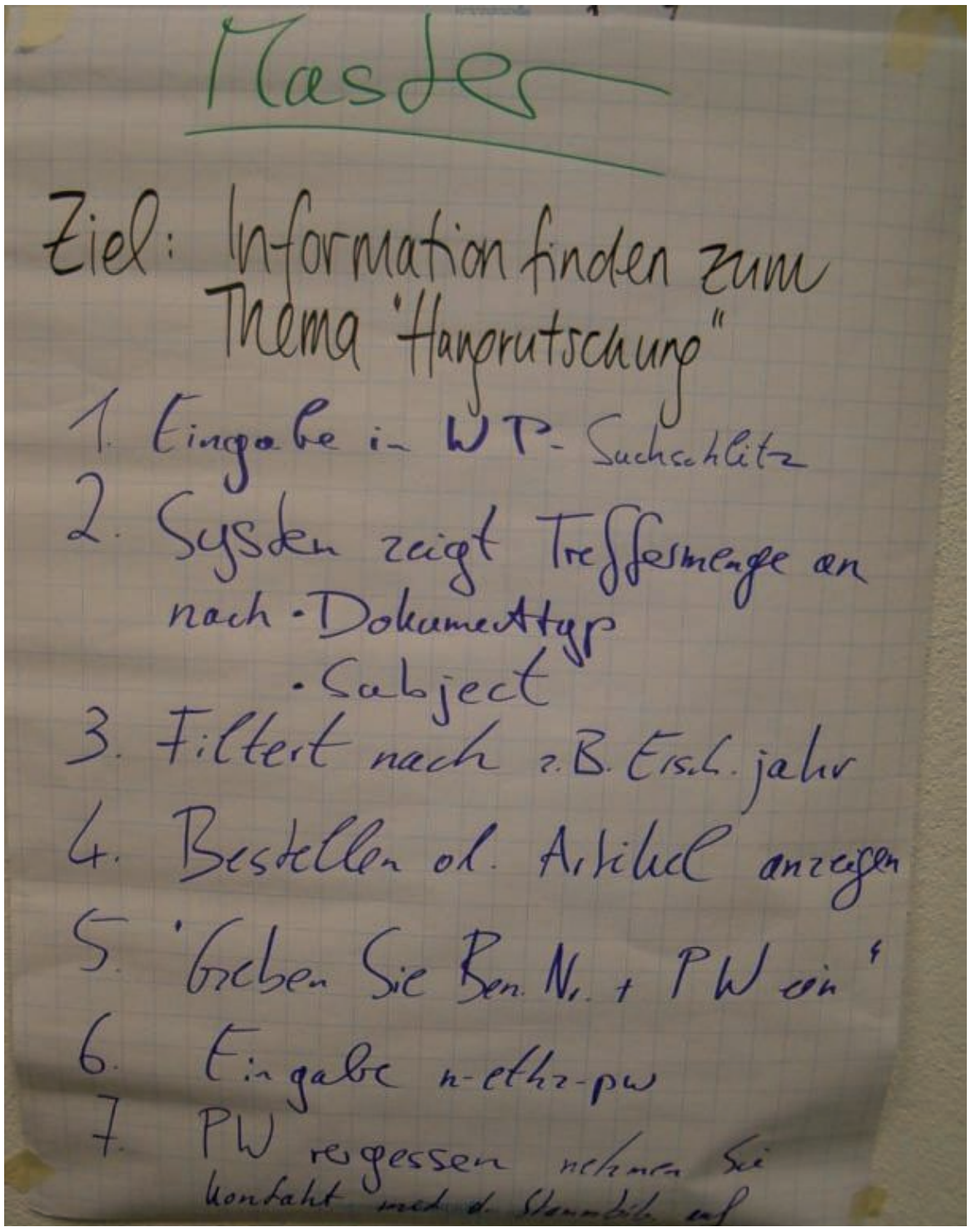

Abb. 3: Benutzerszenario für einen Masterstudierenden oder einen Jungforscher; Skizze aus dem Workshop (Punkt 7: PW vergessen - nehmen Sie Kontakt mit der Stammbibliothek auf) 


\section{Workshop mit Nutzerbeteiligung (Szenarien/Prototyping)}

Um die Website nutzerspezifisch zu gestalten sind einige grundlegende Kenntnisse zum Benutzerverhalten essenziell: Welche Informationen suchen die identifizierten Nutzer über ein zentrales Rechercheinstrument? Was erwarten sie von der Website? Wie geht der Nutzer vor, was sind seine Überlegungen, um die Rechercheaufgabe zu lösen? Welche Funktionalitäten müssen angeboten werden, damit der Nutzer die relevanten Informationen auch auf der Bibliotheks-Website effizient findet?

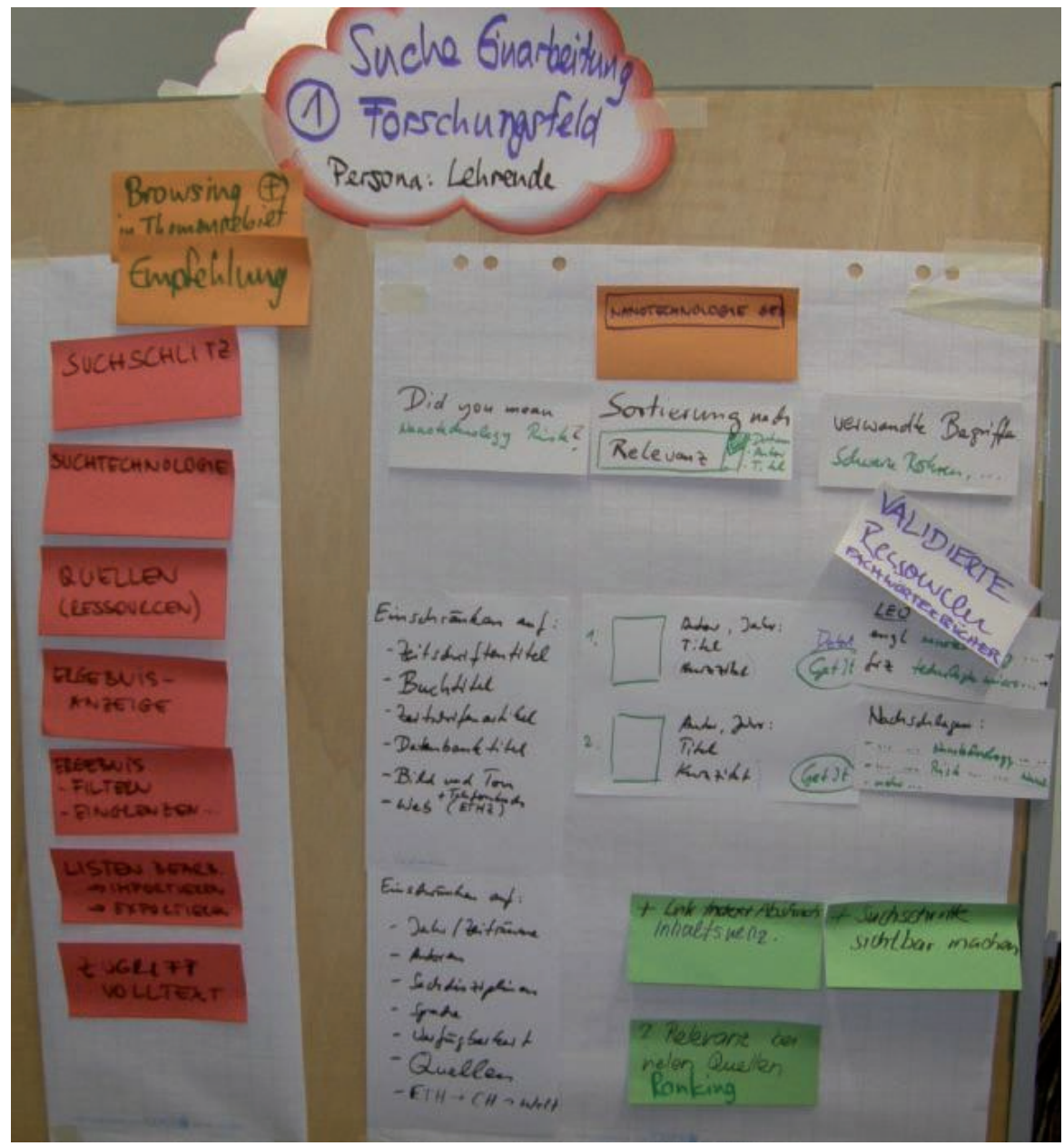

Abb. 4: Erster LoFi ${ }^{14}$-Prototyp für erfahrende Forschende; Szenario: Neue Forschung Nanotechnologie; Skizze aus dem Workshop

14 LoFi: Low Fidelity. 
Um zumindest einige Antworten auf diese Fragen zu erhalten wurde ein zweiter Workshop durchgeführt, diesmal mit repräsentativen Nutzervertretern der Hochschule. Es handelte sich um fünf Benutzer aus unterschiedlichen Fachgebieten und Positionen im Wissenschaftsbetrieb der ETH-Community. Die Beschränkung auf diese ETH-internen Nutzergruppen war gerechtfertigt, da sie die wichtigsten Kunden des zukünftigen Wissensportals sein werden ${ }^{15}$. Der frühe Einbezug der wichtigsten Endnutzer sollte letztlich auch dazu beitragen, die Akzeptanz des Endproduktes zu erhöhen. Ziel des Workshops war es auch, erste Schritte in Richtung inhaltlicher Struktur, Navigationskonzept und PapierPrototypen anzugehen. Mit dem Einbezug einer Außensicht sollten folglich wichtige Erkenntnisse zur zukünftigen Benutzerführung gewonnen werden.

Auch an diesem Workshop wurde lebhaft diskutiert und die Ergebnisse waren auch hier entsprechend ertragreich. Zusammengefasst konnte bestätigt werden, dass die Nutzer einen möglichst einfachen zentralen Zugang zu möglichst vielen Informationsquellen wünschen. Erste wichtige Grundelemente für das zukünftige Wissensportal konnten also bereits identifiziert werden, wobei der zentrale Sucheinstieg ${ }^{16}$ das wichtigste Element war.

\section{Testen der Funktionalitäten des Suchinstruments Primo}

Um auch Erkenntnisse hinsichtlich des Umgangs mit dem Suchinstrument Primo zu erhalten, testeten insgesamt 20 ETH-Angehörige (13 Forschende und Lehrende aus allen Fachgebieten, drei Studierende sowie vier Verwaltungsmitarbeitende) die Funktionalitäten.

Die Mitglieder dieses sogenannten Sounding Boards erhielten einen Link zur Testinstallation von Primo im „Rohzustand ${ }^{17 \text { “ }}$ und einen Link zum OnlineFragebogen. Dieser führte durch die Testaufgaben und forderte die SoundingBoard-Mitglieder auf, diverse Aspekte des Suchinstruments auszuprobieren und die Erfahrungen in einem Fragebogen quantitativ und qualitativ festzuhalten.

15 Die weiteren Nutzergruppen wurden nie aus den Augen verloren: In den Diskussionen wurde ihr erwartetes Verhalten immer wieder angesprochen, vor allem hinsichtlich der einfachen Nutzung.

16 Intensiv wurde auch das Thema Browsing diskutiert mit dem Ergebnis, dass zum Beispiel Literaturlisten von Professoren ein Zugang zu relevanten Informationen sein könnten - viel eher als ein Zugang über thematische Browsinglisten. Man einigte sich schließlich darauf, zuerst die Suche zu realisieren und das umfassende Browsing in einer späteren Version anzugehen.

17 Um die Nutzer auf die wesentlichen Funktionalitäten zu lenken wurden alle nicht relevanten Informationen und Links aus dem Frontend entfernt. 


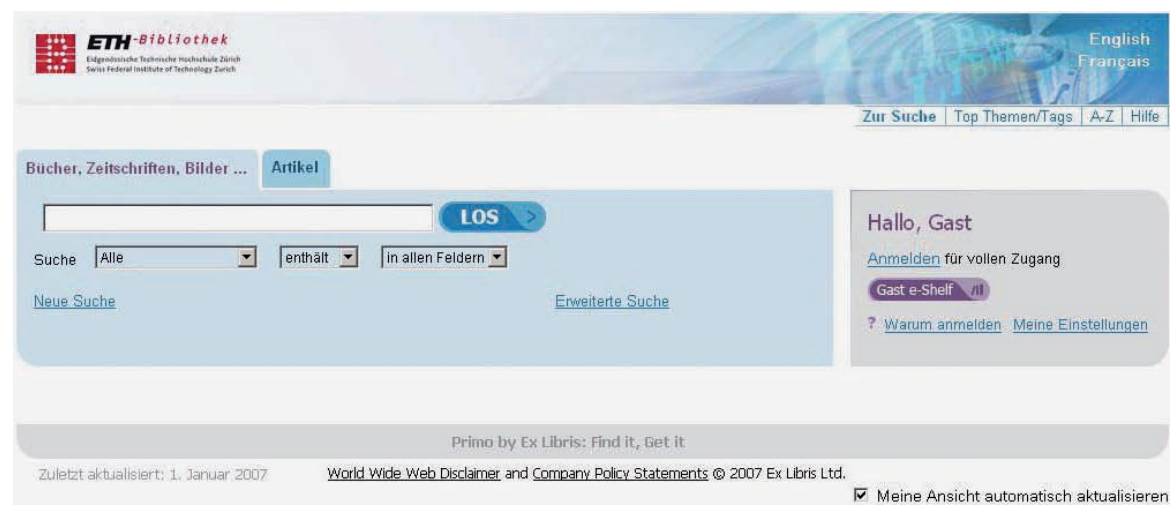

Abb. 5: Screenshot des Suchinstruments im „Rohzustand“

Gefragt wurde nach der Zufriedenheit mit der Suche, den Ergebnissen sowie den Einschränkungsmöglichkeiten von Suchresultaten. Hierzu wurden die Benutzer gebeten, eigene Suchen über die Reiter „Bücher, Zeitschriften, Bilder ... ${ }^{18}$ “ sowie „Artikel ${ }^{19 ،}$ zu tätigen und die angebotenen Funktionalitäten mit Suchanfragen aus ihrem jeweiligen Fachgebiet zu testen. Auch das sogenannte E-Shelf, u.a. mit Funktionen für das Speichern von Suchanfragen, konnte in einem Rohzustand ausprobiert werden. Aufgrund der Ergebnisse wurden mehrere Faktoren identifiziert, die für das definitive Design nützliche Hinweise gaben. Eine wichtige Erkenntnis war, dass auch diejenigen, die das Suchinstrument positiv beurteilten, es als noch nicht bereit für den Launch befanden. So war beispielsweise für manche Testpersonen unklar, welche Informationen sich genau hinter den Reitern versteckten und warum überhaupt zwei Reiter angeboten wurden. Es wurde auch darauf hingewiesen, dass viele angezeigte Informationen bei der eigentlichen Suche gar nicht notwendig sind.

Sehr gelobt wurden die sogenannten Facetten, d.h. extrahierte Suchbegriffe, mit denen eine Ergebnisliste weiter verfeinert werden kann. Eine Testperson fasste ihre Eindrücke wie folgt zusammen: „Es hat einige sehr schöne Innovationen drin. Ein sehr schönes Tool. Es braucht noch Verbesserungen ...". Eine andere Person meinte: „Es hat Spass gemacht und es ist toll, dass das Bibliotheksteam ganz nah an der Benutzerfreundlichkeit dran ist! Ganz großes Kompliment!“

Aufgeteilt nach Personengruppen zeigt das Gesamturteil, dass vor allem jüngere Benutzer, also Studierende sowie Doktorierende, sehr gute Noten austeilten. Es stellt sich hierbei natürlich die Frage, ob diese Kundschaft weniger anspruchsvoll ist oder aber vertrauter mit einem Suchinstrument.

18 Hinter diesem Reiter versteckte sich damals ein Auszug aus dem Katalog NEBIS sowie die Metadaten der ETH E-Collection sowie der Bilddatenbank.

19 Hinter diesem Reiter versteckt sich die eingangs erwähnte Datenbank „DADS“. 
Das Projektteam analysierte sämtliche Rückmeldungen und integrierte sie - wo möglich und sinnvoll - in den Entwicklungsprozess der neuen Website. Es stand insofern vor der günstigen Ausgangslage, als die Einbettung von Primo in die neue Website die Chance bot, noch substanzielle Verbesserungen an der Präsentation der Funktionen und deren Benennung sowie an der Benutzerführung anzubringen.

\section{Herstellung von Papierprototypen}

Die umfangreichen Ergebnisse der Workshops wurden detailliert analysiert. Darauf aufbauend wurden Papierprototypen, also erste Entwürfe der Website, erarbeitet. Bei diesen sogenannten LoFi-Prototypen handelt es sich um primitive Prototypen, häufig erst skizziert auf einem Blatt Papier. Ein Vorteil ist, dass sie kostengünstig und schnell erarbeitet werden können. Damit können bereits in einem frühen Stadium beispielsweise grobe Designfehler eruiert werden, ohne die Website bereits programmiert zu haben.

Bei der Erarbeitung der Prototypen für das Wissensportal hatte das DefaultLayout von Primo selbstverständlich einen gewissen Einfluss. Dies war insofern gerechtfertigt, als die Firma ExLibris die Usability ebenfalls hoch angesetzt hatte und von Benutzern testen ließ. Um die Usability mit der Website der ETH-Bibliothek abzustimmen, musste das Projektteam gewisse Anpassungen des Default-Layouts vornehmen. Hierzu hatten ja die Mitglieder des Sounding Boards wichtige Rückmeldungen gegeben.

Bereits früh im Entwicklungsprozess wurde die Entscheidung getroffen, zunächst die Grundstruktur mit den ersten drei bis vier Ebenen zu planen und erst dann die detaillierten Inhalte nach und nach zu erarbeiten. Ziel war die Herstellung von zwei bis drei Extremvarianten in Form von Storyboards, die dann in einem anschließenden Walkthrough ${ }^{20}$ einigen ausgewählten Nutzern vorgelegt werden sollten. Dadurch sollten den Testpersonen nicht nur das vorgeschlagene Design präsentiert, sondern auch Meinungen provoziert werden.

Als Grundlage für die Erstellung der Prototypen wurde folgender 10-PunktePlan definiert ${ }^{21}$ :

1. Die Suchmaschine steht im Mittelpunkt und ist möglichst auf jeder Seite präsent, sowohl grafisch als auch vom Zugang her. Auch Inhalte/Informationen aus der Website sind mit der zentralen Suche auffindbar und

20 Als Walkthrough bezeichnet man die Analyse des schrittweisen Vorgehens für einen Handlungsablauf, beispielsweise für das Auffinden einer Information auf einer Website.

21 Zu diesem Zeitpunkt war die Evaluation des zukünftigen CMS noch nicht abgeschlossen, die grobe Richtung aber bereits bekannt. 
werden auf der Ergebnisseite gesondert von den Bibliotheksressourcen ausgewiesen.

2. Das Design ist modern, ansprechend und einladend. Grafisches Browsing ersetzt althergebrachte Kategorien und Herangehensweisen.

3. Die Website ist schlank und konzentriert sich auf die wichtigsten Funktionen.

4. Die Struktur ist flexibel, Module/Gadgets sind austauschbar.

5. Alle Arten von möglichen Einstiegen in die Website werden berücksichtigt (über die Homepage, über Suchmaschinen etc.).

6. Das Corporate Design ist immer präsent.

7. Die Aktualität ist gewährleistet: Aktuellmeldungen, neueste Termine u.a.

8. Allenfalls grafischer Zugang für Ausstellungen u.a.

9. Das Layout ist professionell erstellt.

10. Die Inhalte sind professionell redigiert, lektoriert und übersetzt.

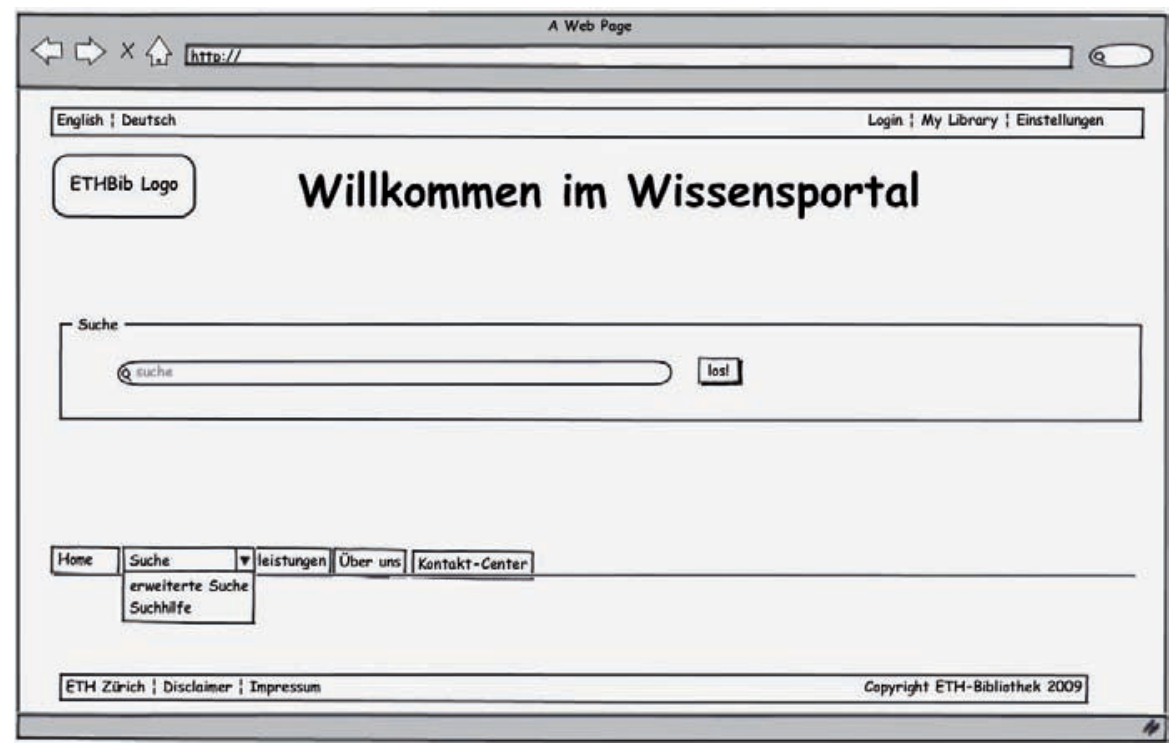

Abb. 6: LoFi-Prototyp eines möglichen Eingangsbildschirms

In einem ersten Schritt wurden die Hauptmenüpunkte auf der ersten Ebene der Website und darauf basierend erste Unterebenen der Sitestruktur festgelegt. Es sollte eine gute Ausgeglichenheit zwischen Auswahlmöglichkeiten auf jeder einzelnen Seite und der Tiefe der Sitestruktur erzielt werden.

In Abbildung 6 ist der LoFi-Prototyp eines möglichen Einstiegsbildschirms dargestellt, in Abbildung 7 derjenige einer erweiterten Suche. Direkte Links zu den wichtigsten Dienstleistungen, sogenannte Quick-Links, wurden bereits zu diesem Zeitpunkt für die Startseite sowie alle Unterseiten diskutiert (vgl. hierzu im unteren Teil der Abb. 7). 
Das Wissensportal der ETH-Bibliothek - ein Fallbeispiel für User-Centered-Design 133

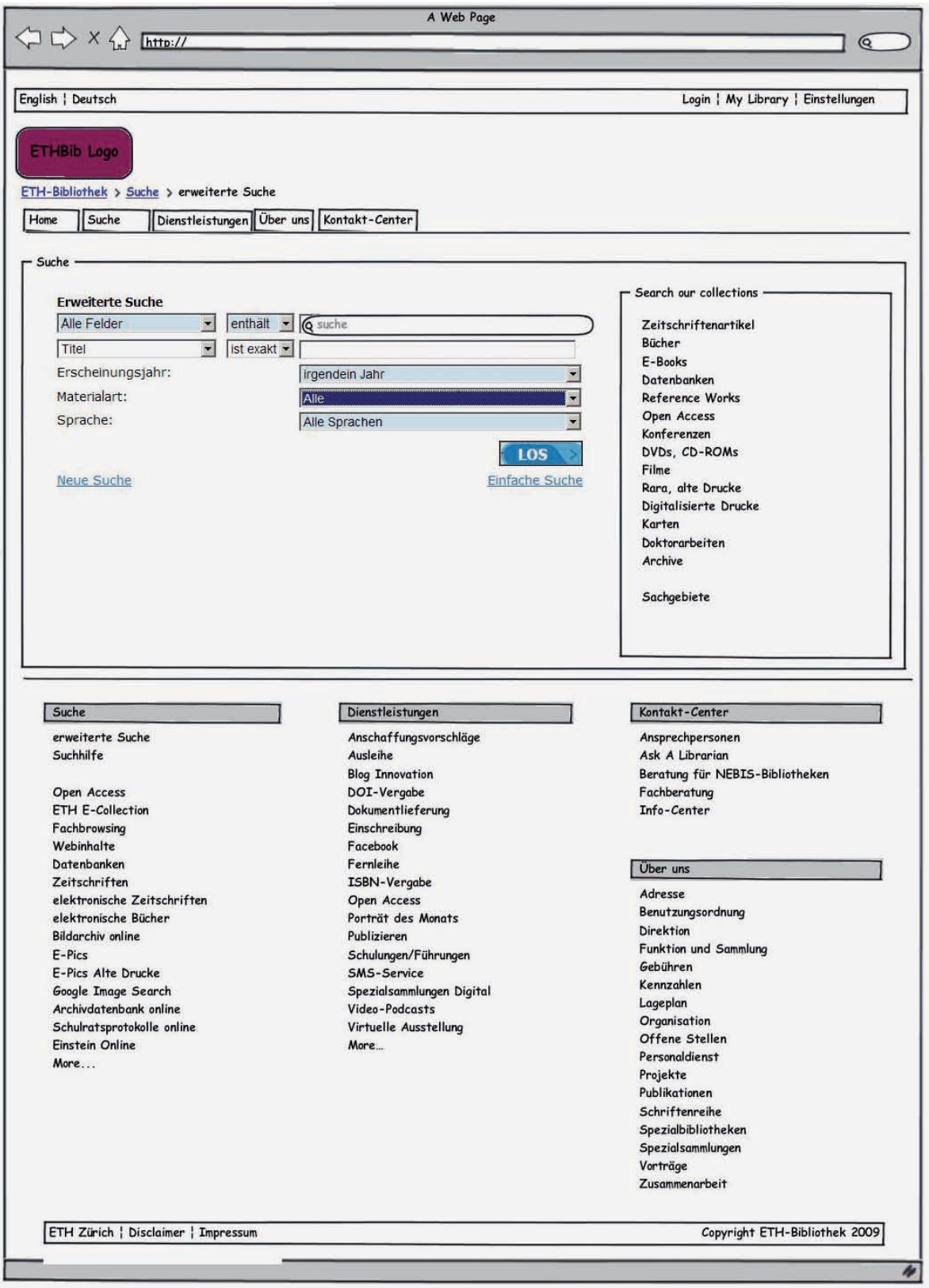

Abb. 7: LoFi-Prototyp eines möglichen Layouts für die erweiterte Suche 


\section{Walkthrough mit Benutzern}

Der nächste Meilenstein war auf Basis der LoFi-Prototypen der Walkthrough mit vier Forschenden ${ }^{22}$ und Lehrenden sowie mit zwei Studierenden. Mit dem Walkthrough wurde das Benutzerverhalten im Umgang mit der zukünftigen Website abgefragt, indem den Probanden verschiedene Szenarien präsentiert wurden, u.a. Startseitensituation, allgemeines Informationsthema (beispielsweise Öffnungszeiten), Suche nach einem bestimmtem Medium, Themensuche, Suche nach Medientyp, Beratungsszenario oder Up-to-date-Bleiben.

Jeder der sechs Probanden wurde vom externen Usability-Experten während 1,5 bis 2 Stunden gezielt befragt, das Gespräch wurde protokolliert. Zwei Projektmitarbeiter verfolgten das Geschehen im Hintergrund.

Die Gespräche waren aufwändig, aber sehr aufschlussreich. Eine kleine Auswahl wichtiger Erkenntnisse ist nachfolgend exemplarisch zusammengefasst:

- Einige Begriffe und Bezeichnungen sind unklar, z.B. InfoCenter, Ausleihe, Datenbanken, Einschreibung, Tagging.

- Einige Begriffe sind zu wenig definiert, z.B. Ausleihe: ist das online? Kann ich hier Bücher vorbestellen? Ist das ein Schalter? Wenn ja, wo ist er?

- Insgesamt zu viele Informationen - lieber wenige und selbsterklärende Angebote

- Hinweise auf die Zugänglichkeit von Medien wären wichtig

- Einstieg über Subjects (Fachbrowsing) viel zu schwierig. Man ist sich nicht im Klaren darüber, was man dabei machen soll. Ist es ein Einstieg, ist es ein Eingrenzen der Suche... wählt man dann mehrere Fachgebiete? Oder nur eines...

- Suchformulare sind zu lang, zu unübersichtlich.

Im Rahmen eines dritten internen Workshops wurden die Ergebnisse dem Projektteam präsentiert und die wichtigsten Erkenntnisse für das Erzielen einer möglichst optimalen Usability der neuen Website festgehalten. Die definitiv festgelegte Struktur bildete - zusammen mit dem erarbeiteten Anforderungskatalog an das Design - dann die Grundlage für den Auftrag an eine WebdesignFirma.

\section{Auftrag an den Webdesigner}

Der Schritt vom festgelegten Konzept zum definitiven Layout ist vermutlich der schwierigste. Trotz umfangreichen Vorgesprächen kann davon ausgegangen werden, dass die Vorstellungen des Auftraggebers wie auch der Projektverantwortlichen in diesem Projektstadium noch weit auseinanderliegen.

22 Von den vier Forschenden waren drei bereits am Workshop beteiligt. 
Um für die ETH-Bibliothek das passende Design zu finden, entschied sich das Projektteam für eine Ausschreibung mit definiertem Profil unter professionellen Webdesignern. Sechs Web-Agenturen folgten der Einladung für die Präsentation der Anforderungen. Diese erhielten alle Agenturen auch in schriftlicher Form und mussten innerhalb zwei Wochen ihre Vorschläge einreichen ${ }^{23}$.

Unter den Bewerbern fanden sich kreative Ideen. Das klar strukturierte Konzept der Firma Hinderling Volkart $\mathrm{AG}^{24}$ traf die Vorstellungen der ETHBibliothek am besten. Es gelang dem Unternehmen unter Berücksichtigung der Vorgaben ein funktional überzeugendes Design für den Webauftritt einer modernen Informationseinrichtung zu entwerfen.

Die Siegerfirma erarbeitete die Detailgestaltung der einzelnen Seitentypen in enger Zusammenarbeit mit dem für die Website zuständigen ProjektSubteam. Obwohl im Hintergrund zwei ganz verschiedene Systeme stehen einerseits das CMS eZ Publish für die Verwaltung der Inhalte der Website und andererseits das Frontend von Primo - musste sich der neue Webauftritt der ETH-Bibliothek aus einem Guss präsentieren. In diesem Prozess war vor allem die Detailgestaltung verhältnismässig langwierig. Zwischen den Technikern und den Bibliothekaren herrschten häufig unterschiedliche Meinungen hinsichtlich der Gestaltung und Positionierung einzelner Elemente. Mit einigen Kompromissen gelang es letztlich aber sehr überzeugend, die Informationsarchitektur, das Navigationskonzept sowie das Layout von Primo und CMS nahtlos ineinandergreifend zu präsentieren - selbstverständlich unter Berücksichtigung der gewonnenen Nutzerbedürfnisse.

Das Visual-Design wurde anschließend von der Web-Agentur in HTML/ CSS Files umgesetzt. Basierend auf diesen Files wurden die eZ Publish Templates für das Content Management System (CMS) entwickelt ${ }^{25}$.

\section{Erarbeitung der künftigen Inhalte im erweiterten Webteam}

Die Erkenntnisse aus dem User-Centered-Design-Prozess bildete auch eine wichtige Grundlage für die Erarbeitung der Inhalte der Website in Absprache mit den fachlich jeweils zuständigen Bereichen der ETH-Bibliothek. Um einen einheitlichen Aufbau der einzelnen Websites zu erzielen wurde je eine Grundstruktur festgelegt. Grundlage für die Texte bildeten darüber hinaus auch die „Leitlinien für die Gestaltung von Grafik und Texten in der ETH-Bibliothek ${ }^{26 ، “}$.

23 Da das Design der Website der Hochschule bereits in die Jahre gekommen war, aber eine Erneuerung noch nicht konkretisiert war, entschied sich die ETH-Bibliothek für das Aufsetzen eines eigenen Designs. Selbstverständlich wurden die Auflagen des Bundes für behindertengerechte Websites berücksichtigt.

24 Vgl. hierzu: http://www.hinderlingvolkart.com.

25 Die CMS-Vorlagen von eZ Publish wurden von einer externen Firma erstellt.

26 Das Dokument dient als Grundlage für Marketing und Kommunikation in der ETHBibliothek. 
Ein kleines Redaktionsteam bereitete die Texte auf Basis von formalen Vorgaben auf. Die damals auf den aktuellen Websites der ETH-Bibliothek vorhandenen Informationen wurden neu strukturiert, teilweise gekürzt oder ergänzt bzw. meist ganz neu geschrieben. Das Einfüllen in sogenannte Mock-Ups erlaubte eine einfache Überarbeitung der Entwürfe von diversen Mitarbeitenden. Die Titel der Seiten wurden so gewählt, dass sie die Navigation unterstützen. Für die Schlussbearbeitung der Texte wurde ein externes Lektorat beauftragt. Die Übersetzung ins Englische wurde von einem Übersetzungsbüro vorgenommen. Anhand eines erarbeiteten Styleguides, der die Grundlagen der visuellen und typographischen Regeln für die Website vorgibt, wurden dann die vorbereiteten Inhalte in das CMS eingepflegt.

\section{Projektergebnis und Fazit}

Das Wissensportal wurde im Sommer 2010 erfolgreich eingeführt, begleitet von einigen hochschulweiten Marketingmassnahmen. Das Portal präsentiert sich heute wie folgt (vgl. hierzu http://www.library.ethz.ch und Abb. 8):

- Die Website gliedert sich in die Hauptgruppen Home, Dienstleistungen, Ressourcen, Über uns, Kontakt.

- Das Suchfenster ist auf jeder Seite prominent platziert.

- Das Suchinstrument Primo ist integriert in die Website mit einem einheitlichen, funktionalen und attraktiven Layout.

- Die integrierten Informationsressourcen sind mit einer Suchanfrage durchsuchbar (Katalog, Institutional Repository, Bilddatenbank etc.). Der Zugriff auf die elektronischen Volltexte erfolgt nahtlos über einen Link.

- Die Informationen zu allen Dienstleistungen der ETH-Bibliothek sind klar strukturiert und in einer verständlichen Sprache verfügbar.

- Links zu den wichtigsten Dienstleistungen werden als sogenannte Quicklinks auf der Haupt- und allen Unterseiten aufgeführt.

Mit dem neuen Wissensportal unterstreicht die ETH-Bibliothek ihren Anspruch als modernes Informationszentrum. Mit der nahtlosen Integration von Primo in die Website mit einem eigenen attraktiven Design hat sie Pionierarbeit geleistet. Darüber hinaus hat sie mit dem frühen Einbezug der wichtigsten Zielgruppen und einem zeitaufwändigen User-Centered-Design-Prozess ein Produkt geschaffen, das von der wissenschaftlichen Community der ETH Zürich wie auch von den weiteren Zielgruppen breit akzeptiert ist. Die zentrale und einfache Suche über mehrere Ressourcen hinweg wird von einer großen Kundschaft sehr geschätzt. Einzelne kleinere Unzulänglichkeiten werden laufend verbessert.

Als Fazit kann festgehalten werden, dass sich das zeit- und ressourcenintensive Verfahren gelohnt hat. Als Kritik mag angefügt werden, dass das schließlich 


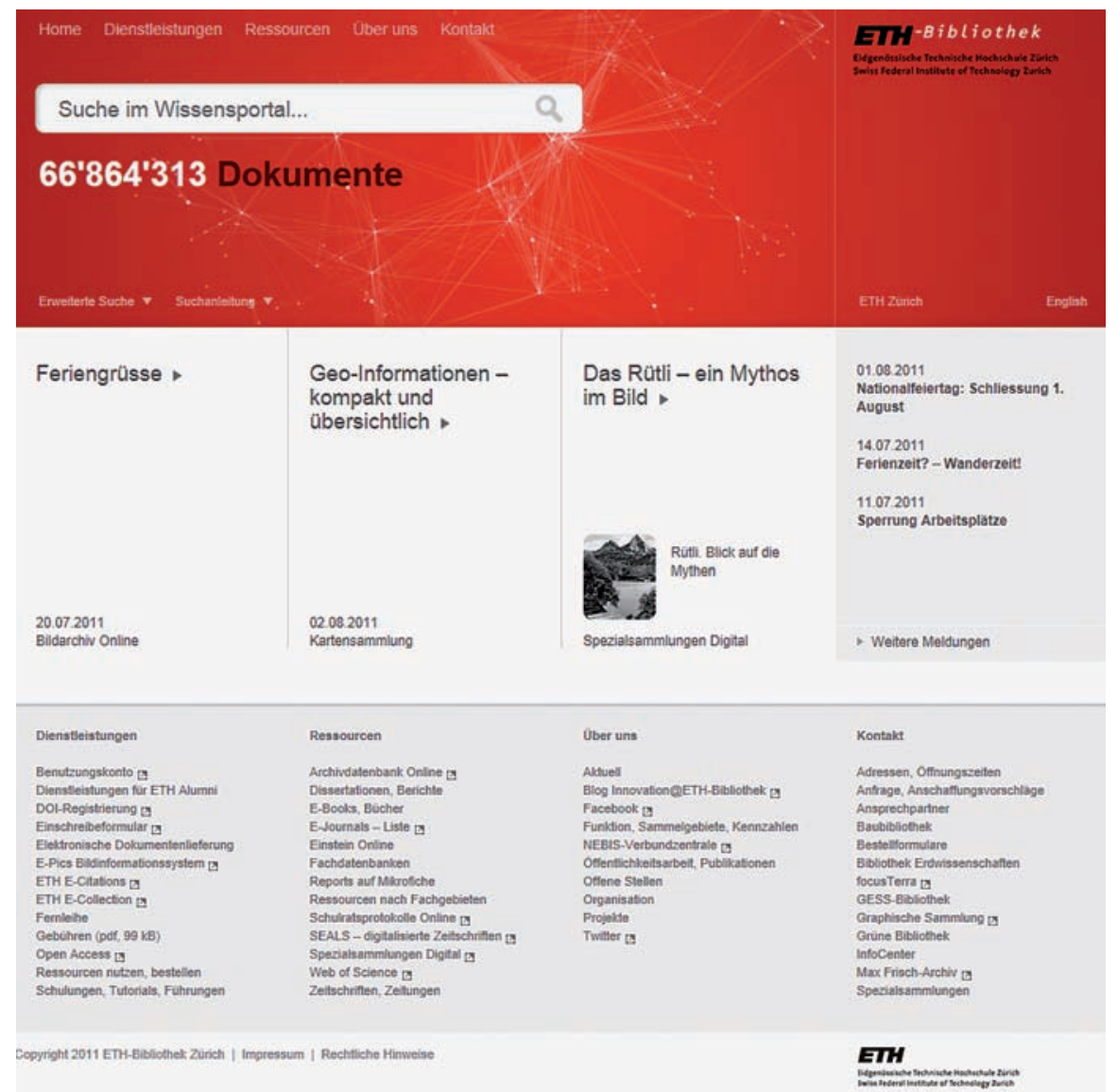

Abb. 8: Aktuelle Homepage der ETH-Bibliothek

erstellte Design vor dem Aufschalten nicht mehr mit externen Nutzern getestet und adaptiert wurde. Dies hat mehrere Gründe: Einerseits wäre die Projektlaufzeit massiv überschritten worden, andererseits konnte die erfahrene Firma für das Webdesign wichtige Funktionalitäten und Darstellungen fundiert empfehlen.

Möglich wäre nun die Durchführung eines heuristischen Verfahrens für die Evaluation der Usability, um mögliche Schwachstellen der Website zu ermitteln. Hierbei würde eine kleine Gruppe von Experten die Benutzerschnittstelle des Portals analysieren und die Übereinstimmung mit bestimmten Usability Prinzipien (Heuristiken) überprüfen. Ob und wann diese Evaluation durchgeführt wird, ist noch offen.

Um das Wissensportal attraktiv zu halten, sollen laufend neue Informationsquellen integriert und weitere Funktionalitäten realisiert werden. Ein wichtiger Schritt wird hierbei die Einführung des Single-Sign-on sein, mit dem sich ins- 
besondere für Angehörige der ETH Zürich das mehrfache Anmelden bei verschiedenen Informationsquellen erübrigen wird. Hinzu kommen die Personalisierung des Angebots, indem beispielsweise die Resultate von Abfragen gespeichert werden können, sowie die Nutzung des Wissensportals über mobile Geräte. Es wird eine Herausforderung sein, diese Funktionalitäten so in die bestehende Website einzufügen, dass die Usability weiterhin einfach und intuitiv bleibt. Auf die wertvolle Mitarbeit der Benutzer wird die ETH-Bibliothek auch hier sicher nicht verzichten!

\section{Literatur}

Cooper, A. (1999): The inmates are running the asylum. Macmillan: Indianapolis.

Gould D.J. und Lewis C. (1985): Designing for Usability: Key Principles and What Designers Think. Communications of the ACM, 28(3), S. 300-311.

Hegner, M. (2003): Methoden zur Evaluation von Software. IZ Arbeitsbericht Nr. 29. http:// www.gesis.org/fileadmin/upload/forschung/publikationen/gesis_reihen/iz_arbeitsberichte/ ab_29.pdf (14.07.2011).

Jordan, P.W. (1998): An Introduction to usability, London: Taylor and Francis.

Normann, D. (1998): The Invisible Computer. Cambridge MA, MIT Press.

Ramminger E. und Graf, N. (2007): Informationsmanagement an der ETH Zürich. Ergebnisse einer qualitativen Expertenbefragung der ETH-Bibliothek zum Umgang mit Literatur und Informationsressourcen in Forschung und Lehre ETH-Bibliothek. doi:10.3929/ ethz-a-005472952.

useit.com: Jakob Nielsen's Website. http://www.useit.com/ (13.07.2011). 